Proceedings of the Edinburgh Mathematical Society (2005) 48, 557-569 (C)

DOI:10.1017/S0013091504000549 Printed in the United Kingdom

\title{
THE RELATIVE PICARD GROUP OF A COMODULE ALGEBRA AND HARRISON COHOMOLOGY
}

\author{
S. CAENEPEEL AND T. GUÉDÉNON \\ Faculty of Engineering, Vrije Universiteit Brussel, B-1050 Brussels, Belgium \\ (scaenepe@vub.ac.be; tguedeno@vub.ac.be)
}

(Received 20 April 2004)

\begin{abstract}
Let $A$ be a commutative comodule algebra over a commutative bialgebra $H$. The group of invertible relative Hopf modules maps to the Picard group of $A$, and the kernel is described as a quotient group of the group of invertible group-like elements of the coring $A \otimes H$, or as a Harrison cohomology group. Our methods are based on elementary $K$-theory. The Hilbert 90 theorem follows as a corollary. The part of the Picard group of the coinvariants that becomes trivial after base extension embeds in the Harrison cohomology group, and the image is contained in a well-defined subgroup $E$. It equals $E$ if $H$ is a cosemisimple Hopf algebra over a field.
\end{abstract}

Keywords: Picard group; coring; Harrison cohomology

2000 Mathematics subject classification: Primary 16W30

\section{Introduction}

Let $l$ be a cyclic Galois field extension of $k$. The Hilbert 90 theorem tells us that every cocycle in $Z^{1}\left(C_{p}, l^{*}\right)$ is a coboundary. There exist various generalizations of this result. For example, if we have a Galois extension $B \rightarrow A$ of commutative rings, with Galois group $G$, then the cohomology group $H^{1}\left(G, \mathbb{G}_{m}(A)\right)$ is isomorphic to $\operatorname{Pic}(A / B)$, the kernel of the natural map from the Picard group of $B$ to the Picard group of $A$ (see, for example, $[\mathbf{9}])$. Now we can ask the following question. Suppose that $G$ acts on $A$ as a group of isomorphisms. Can we still give an algebraic interpretation of $H^{1}\left(G, \mathbb{G}_{m}(A)\right)$ ? A second problem is whether there is any relation between $H^{1}\left(G, \mathbb{G}_{m}(A)\right)$ and the Picard group of the ring of invariants $B=A^{G}$.

In this paper we will discuss these two problems in a more general situation: we will assume that $A$ is a commutative $H$-comodule algebra, with $H$ an arbitrary commutative bialgebra over a commutative ring $k$. We then ask for an algebraic interpretation of the first Harrison cohomology group $H_{\mathrm{Harr}}^{1}\left(H, A, \mathbb{G}_{m}\right)$ (with notation as in [6]). If $H$ is finitely generated and projective, then this Harrison cohomology group is isomorphic to a Sweedler cohomology group $Z_{\text {Harr }}^{1}\left(H, A, \mathbb{G}_{m}\right)$, and if $H=\mathbb{Z} G$ with $G$ a finite group, then it reduces to the cohomology group $H^{1}\left(G, \mathbb{G}_{m}(A)\right)$.

We proceed as follows: we introduce the relative Picard group $\operatorname{Pic}^{H}(A)$ as the Grothendieck group of the category of invertible relative Hopf modules. The forgetful 
functor to the category of invertible $A$-modules induces a $K$-theoretic exact sequence, linking the Picard group of $A$, the relative Picard group, and the groups of unit elements of $A$ and the coinvariants $B=A^{\text {co } H}$; the middle term in the sequence can be computed, and it is the group of invertible group-like elements of the coring $A \otimes H$. We show also that these group-like elements are precisely the Harrison cocycles, and it follows from the exactness of the sequence that the first Harrison cohomology group is the kernel of the map $\operatorname{Pic}^{H}(A) \rightarrow \operatorname{Pic}(A)$, answering our first question.

Then we observe that there is a similar exact sequence associated with the induction functor $\operatorname{Pic}(B) \rightarrow \underline{\operatorname{Pic}}(A)$, and that the two exact sequences fit into a commutative

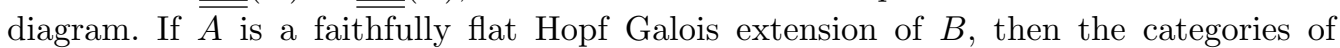
$B$-modules and relative Hopf modules are equivalent, hence $\operatorname{Pic}(B) \cong \operatorname{Pic}^{H}(A)$, and we recover Hilbert 90. In general, we have an injection $\operatorname{Pic}(A / B) \rightarrow H_{\mathrm{Harr}}^{1}\left(H, A, \mathbb{G}_{m}\right)$, and we can describe a subgroup of $H_{\mathrm{Harr}}^{1}\left(H, A, \mathbb{G}_{m}\right)$ that contains the image of $\operatorname{Pic}(A / B)$. The image is precisely this subgroup if $H$ is a cosemisimple Hopf algebra over a field $k$.

A special situation is the following: let $k$ be an algebraically closed field, $A$ a finitely generated commutative normal $k$-algebra, and $G$ a connected algebraic group acting rationally on $A$. Then $A$ is an $H$-comodule algebra, with $H$ the affine coordinate ring of $G$. In this case, our exact sequence was given by Magid in [14], but apparently Magid was not aware of the connection to Harrison cohomology, group-like elements of corings or the generalized Hilbert 90 theorem.

In $\S 5$, we study the Harrison cocycles (or the group-like elements in $A \otimes H$ ) in some particular cases. First we look at the situation considered by Magid in [14], and then it turns out that the group-like elements of $G(A \otimes H)$ are induced by the group-like elements of $H$. In the situation where $A$ is a $\mathbb{Z}$-graded commutative $k$-algebra, the relative Picard group turns out to be the graded Picard group $\operatorname{Pic}_{\mathrm{g}}(A)$ studied by the first author in [5]. If $A$ is reduced, then the group-like elements of $A \otimes H$ can also be described using the group-like elements of $H$, according to a result in [5].

\section{Preliminary results}

\subsection{The language of corings}

Relative Hopf modules can be viewed as comodules over a coring. This will be used below, and this is why we briefly recall some properties of corings. Recall that an $A$-coring is a comonoid in the monoidal category ${ }_{A} \mathcal{M}_{A}$ of $A$-bimodules. Thus an $A$-coring $\mathfrak{C}$ is an $A$-bimodule together with two $A$-bimodule maps,

$$
\Delta_{\mathfrak{C}}: \mathfrak{C} \rightarrow \mathfrak{C} \otimes_{A} \mathfrak{C} \quad \text { and } \quad \varepsilon_{\mathfrak{C}}: \mathfrak{C} \rightarrow A,
$$

satisfying the usual coassociativity and counit properties. We refer to $[\mathbf{2 - 4 , 1 1 , 1 8}]$ for a detailed discussion of corings. The set of group-like elements of $\mathfrak{C}$ is given by

$$
G(\mathfrak{C})=\left\{X \in \mathfrak{C} \mid \Delta_{\mathfrak{C}}(X)=X \otimes_{A} X \text { and } \varepsilon_{\mathfrak{C}}(X)=1\right\} .
$$


A right $\mathfrak{C}$-comodule $M$ is a right $A$-module together with a right $A$-linear map $\rho_{M}: M \rightarrow$ $M \otimes_{A} \mathfrak{C}$ satisfying

$$
\left(M \otimes_{A} \varepsilon_{\mathfrak{C}}\right) \circ \rho_{M}=M \quad \text { and } \quad\left(M \otimes_{A} \Delta_{\mathfrak{C}}\right) \circ \rho_{M}=\left(\rho_{M} \otimes_{A} \mathfrak{C}\right) \circ \rho_{M} .
$$

A morphism of right $\mathfrak{C}$-comodules $f: M \rightarrow N$ is an $A$-linear map $f$ such that

$$
\rho_{N} \circ f=\left(f \otimes_{A} \mathfrak{C}\right) \circ \rho_{M} .
$$

$\mathcal{M}^{\mathfrak{C}}$ will be the category of right $\mathfrak{C}$-comodules and comodule morphisms. We have the following interpretation of the group-like elements of $\mathfrak{C}$.

Lemma 2.1. Let $\mathfrak{C}$ be an $A$-coring. Then there is a bijective correspondence between $G(\mathfrak{C})$ and the set of maps $\rho: A \rightarrow A \otimes_{A} \mathfrak{C}=\mathfrak{C}$, making $A$ into a right $\mathfrak{C}$-comodule. The coaction $\rho_{X}$ corresponding to $X \in G(\mathfrak{C})$ is given by

$$
\rho_{X}(a)=X a .
$$

With this notation, $A^{X}=\left(A, \rho_{X}\right)$ is isomorphic to $A^{Y}=\left(A, \rho_{Y}\right)$ as a right $\mathfrak{C}$-comodule if and only if there exists an invertible $b \in A$ such that $\rho_{Y}(b)=Y b=b X$.

Proof. The first part is well known (and straightforward) (see, for example, [3]). Let $f: A^{X} \rightarrow A^{Y}$ be a right $\mathfrak{C}$-colinear isomorphism. Then $f(a)=b a$ for some $b \in A$, which is invertible since $f$ is an isomorphism. The fact that $f$ is $\mathfrak{C}$-colinear tells us that

$$
Y b=\rho_{Y}(f(1))=\left(f \otimes_{A} \mathfrak{C}\right)\left(\rho_{X}(1)\right)=b X .
$$

The converse property is obvious.

\subsection{Relative Hopf modules}

Let $H$ be a bialgebra over a commutative ring $k$, and $A$ a right $H$-comodule algebra. Throughout this paper we will assume that $H$ and $A$ are commutative. Then $A$ is a commutative algebra and we have a right $H$-coaction $\rho$ on $A$ such that

$$
\rho(a b)=a_{[0]} b_{[0]} \otimes a_{[1]} b_{[1]},
$$

for all $a, b \in A$. Here we use the Sweedler-Heyneman notation for the coaction $\rho: \rho(a)=$ $a_{[0]} \otimes a_{[1]}$, with summation implicitly understood. For the comultiplication on $H$, we use the notation $\Delta(h)=h_{(1)} \otimes h_{(2)}$.

A relative Hopf module $M$ is a $k$-module, together with a right $A$-action and a right $C$-coaction $\rho_{M}$ such that

$$
\rho_{M}(m a)=m_{[0]} a_{[0]} \otimes m_{[1]} a_{[1]},
$$

for all $a \in A$ and $m \in M$. The category of relative Hopf modules and $A$-linear $H$-colinear maps will be denoted by $\mathcal{M}_{A}^{H}$. The coinvariant submodule $M^{\text {co } H}$ of $M \in \mathcal{M}_{A}^{H}$ is defined by

$$
M^{\mathrm{co} H}=\left\{m \in M \mid \rho_{M}(m)=m \otimes 1\right\} .
$$


$A^{\text {co } H}=B$ is a $k$-subalgebra of $A$, and $M^{\text {co } H}$ is a $B$-module. We obtain a functor $(\cdot)^{\operatorname{co} H}: \mathcal{M}_{A}^{H} \rightarrow \mathcal{M}_{B}$, which has a left adjoint $T=-\otimes_{B} A: \mathcal{M}_{B} \rightarrow \mathcal{M}_{A}^{H}$. The right $H$-coaction on $N \otimes_{B} A$ is $N \otimes_{B} \rho$. The unit $u$ and counit $c$ of the adjunction are given by the following formulae, for $N \in \mathcal{M}_{B}$ and $M \in \mathcal{M}_{A}^{H}$ :

$$
\begin{array}{ll}
u_{N}: N \rightarrow\left(N \otimes_{B} A\right)^{\text {co } H}, & u_{N}(n)=n \otimes 1, \\
c_{M}: M^{\operatorname{co} H} \otimes_{B} A \rightarrow M, & c_{M}(m \otimes a)=m a .
\end{array}
$$

$A$ is called a Hopf algebra extension of $B=A^{\text {co } H}$ if the canonical map

$$
\text { can }: A \otimes_{B} A \rightarrow A \otimes H, \quad \operatorname{can}\left(a \otimes_{B} b\right)=a b_{[0]} \otimes b_{[1]}
$$

is an isomorphism. If $A$ is a faithfully flat Hopf Galois extension, then the adjunction $\left(-\otimes_{B} A,(\cdot)^{\mathrm{co} H}\right)$ is a pair of inverse equivalences. We refer to $[\mathbf{1 0}, \mathbf{1 5}, \mathbf{1 7}]$ for a detailed discussion of Hopf algebras and relative Hopf modules.

$\mathfrak{C}=A \otimes H$ is a coring, with structure maps

$$
\begin{aligned}
a^{\prime}(b \otimes h) a & =a^{\prime} b a_{[0]} \otimes h a_{[1]}, \\
\Delta_{\mathfrak{C}}(a \otimes h) & =\left(a \otimes h_{(1)}\right) \otimes_{A}\left(1 \otimes h_{(2)}\right), \\
\varepsilon_{\mathfrak{C}}(a \otimes h) & =a \varepsilon(h) .
\end{aligned}
$$

The category $\mathcal{M}^{A \otimes H}$ is isomorphic to the category $\mathcal{M}_{A}^{H}$ of relative Hopf modules; we refer to $[\mathbf{2}, \mathbf{4}]$ for full details. Note that $X=\sum_{i} a_{i} \otimes h_{i} \in G(A \otimes H)$ if and only if

$$
\sum_{i}\left(a_{i} \otimes h_{i(1)} \otimes h_{i(2)}\right)=\sum_{i, j}\left(a_{i} a_{j[0]} \otimes h_{i} a_{j[1]} \otimes h_{j}\right) \quad \text { and } \quad \sum a_{i} \varepsilon\left(h_{i}\right)=1 .
$$

$A \otimes H$ is also a commutative algebra, with multiplication

$$
(a \otimes h)(b \otimes k)=a b \otimes h k .
$$

The product of two group-like elements is a group-like element, and $1_{A} \otimes 1_{H}$ is grouplike. Hence $G^{i}(A \otimes H)$, the set of invertible group-like elements, is an abelian group. Also observe that an invertible group-like element is precisely a normalized Harrison 1-cocycle (see, for example, $[\mathbf{6}, \S 9.2]$ for the definition of the Harrison complex).

Let $H$ be a finitely generated projective cocommutative Hopf algebra, and let $A$ be a commutative left $H$-module algebra. Then $H^{*}$ is a commutative Hopf algebra and $A$ is a right $H^{*}$-comodule algebra. If $\sum_{i} a_{i} \otimes f_{i} \in A \otimes H^{*}$ is an invertible group-like element (or a normalized Harrison cocycle), then

$$
\phi: H \rightarrow A, \quad \phi(h)=\sum_{i} a_{i} f_{i}(h),
$$

is a normalized Sweedler 1-cocycle. This means that $\phi\left(1_{H}\right)=1_{A}$, and the cocycle condition

$$
\phi\left(h h^{\prime}\right)=\sum_{i}\left(h_{(1)} \cdot\left(\phi\left(h^{\prime}\right)\right)\right) \phi\left(h_{(2)}\right)
$$


is satisfied. This gives a bijective correspondence between Harrison and Sweedler cocycles, see [6, Proposition 9.2.3]. For the definition of the Sweedler complex, see [16] or [6, § 9.1]. In the case where $H=k G$, with $G$ a finite group, Sweedler cohomology reduces to group cohomology.

\subsection{Elementary algebraic $K$-theory}

Let $(\mathcal{C}, \otimes, I)$ and $(\mathcal{D}, \otimes, J)$ be skeletally small symmetric monoidal categories, and let $F: \mathcal{C} \rightarrow \mathcal{D}$ be a cofinal, strong monoidal functor. Then we can consider the Grothendieck and Whitehead groups of $\mathcal{C}$ and $\mathcal{D}$, and we have an exact sequence connecting them (see, for example, [1, Chapter VII]):

$$
K_{1} \mathcal{C} \stackrel{K_{1} F}{\longrightarrow} K_{1} \mathcal{D} \stackrel{d}{\rightarrow} K_{1} \underline{\phi F} \stackrel{g}{\rightarrow} K_{0} \mathcal{C} \stackrel{K_{0} F}{\longrightarrow} K_{0} \mathcal{D} .
$$

$C \in \mathcal{C}$ is called invertible if there exists $C^{\prime} \in \mathcal{C}$ such that $C \otimes C^{\prime} \cong I$. If all elements of $\mathcal{C}$ and $\mathcal{D}$ are invertible, then the description of the five groups in (2.4) and the connecting maps simplifies (see $[\mathbf{6}$, Appendix $\mathrm{C}]$ ). $K_{0} \mathcal{C}$ is the group of isomorphism classes of objects in $\mathcal{C}$ and $K_{1} \mathcal{C} \cong \operatorname{Aut}_{\mathcal{C}}(I)$ (which is then an abelian group). Let $\underline{\Psi F}$ be the following category: objects are couples $(C, \alpha)$, with $C \in \mathcal{C}$ and $\alpha: F(C) \rightarrow J$ an isomorphism in $\mathcal{D}$. A morphism between $(C, \alpha)$ and $\left(C^{\prime}, \alpha^{\prime}\right)$ is an isomorphism $f: C \rightarrow C^{\prime}$ in $\mathcal{C}$ such that $\alpha^{\prime}=F(f) \circ \alpha . \underline{\Psi F}$ is monoidal, every object is invertible and

$$
K_{1} \underline{\phi F} \stackrel{g}{\rightarrow} \cong K_{0} \underline{\Psi F} .
$$

The maps $d$ and $g$ are given as follows: $d(\alpha)=[(I, \alpha)]$ and $g[(C, \alpha)]=[C]$.

A typical example is the following: for a commutative ring $A$, let $\underline{\underline{\operatorname{Pic}}}(A)$ be the category of invertible $A$-modules. If $i: B \rightarrow A$ is a morphism of commutative rings, then we have the cofinal strongly monoidal functor

$$
G=-\otimes_{B} A: \underline{\underline{\operatorname{Pic}}}(B) \rightarrow \underline{\underline{\operatorname{Pic}}}(A)
$$

and (2.4) takes the form

$$
1 \rightarrow \mathbb{G}_{m}(B) \rightarrow \mathbb{G}_{m}(A) \stackrel{d^{\prime}}{\rightarrow} K_{1} \underline{\phi G} \stackrel{g^{\prime}}{\rightarrow} \operatorname{Pic}(B) \rightarrow \operatorname{Pic}(A) .
$$

\section{The relative Picard group}

If $M, N \in \mathcal{M}_{A}^{H}$, then $M \otimes_{A} N \in \mathcal{M}_{A}^{H}$, with right $H$-coaction

$$
\rho_{M \otimes_{A} N}\left(m \otimes_{A} n\right)=m_{[0]} \otimes_{A} n_{[0]} \otimes m_{[1]} n_{[1]} .
$$

So we have a symmetric monoidal category $\left(\mathcal{M}_{A}^{H}, \otimes_{A}, A\right)$. Let $\underline{\operatorname{Pic}}^{H}(A)$ be the full subcategory consisting of invertible objects. $\operatorname{Pic}^{H}(A)=K_{0}{\underline{\underline{\mathrm{Pic}^{H}}}}^{H}(A), \overline{\overline{\text { the }}}$ group of isomorphism classes of relative Hopf modules, will be called the relative Picard group of $A$ and $H$. The isomorphism class in $\operatorname{Pic}^{H}(A)$ represented by an invertible relative Hopf module $M$ will be denoted by $\{M\}$. This new invariant fits into an exact sequence. 
Proposition 3.1. We have an exact sequence

$$
1 \rightarrow \mathbb{G}_{m}(B) \rightarrow \mathbb{G}_{m}(A) \stackrel{d}{\rightarrow} G^{i}(A \otimes H) \stackrel{g}{\rightarrow} \operatorname{Pic}^{H}(A) \rightarrow \operatorname{Pic}(A) .
$$

Proof. This result can be proved in two ways: a first possibility is to show that (3.1) is precisely the exact sequence (2.4), associated with the functor $\underline{\mathrm{Pic}}^{H}(A) \rightarrow \underline{\operatorname{Pic}}(A)$ forgetting the $H$-coaction. Let us present an easy direct proof.

The map $\mathbb{G}_{m}(B) \rightarrow \mathbb{G}_{m}(A)$ is the natural inclusion. Take $a \in A$ invertible, and let $d(a)=X=a^{-1} a_{[0]} \otimes a_{[1]} . X$ is group-like, since $a^{-1} a_{[0]} \varepsilon\left(a_{[1]}\right)=1$, and

$$
\begin{aligned}
X \otimes_{A} X & =\left(a^{-1} a_{[0]} \otimes a_{[1]}\right) \otimes_{A}\left(b^{-1} b_{[0]} \otimes b_{[1]}\right) \\
& =a^{-1} a_{[0]}\left(b^{-1}\right)_{[0]} b_{[0]} \otimes a_{[1]}\left(b^{-1}\right)_{[1]} b_{[1]} \otimes b_{[2]} \\
& =a^{-1} b_{[0]} \otimes b_{[1]} \otimes b_{[2]} \\
& =\left(a^{-1} b_{[0]} \otimes b_{[1]}\right) \otimes_{A}\left(1 \otimes b_{[2]}\right)=\Delta(X),
\end{aligned}
$$

where we identified $(A \otimes H) \otimes_{A}(A \otimes H)=A \otimes H \otimes H$ and we wrote $a=b$. The inverse of $X$ is $X^{-1}=a\left(a^{-1}\right)_{[0]} \otimes\left(a^{-1}\right)_{[1]}$, so $X \in G^{i}(A \otimes H)$.

If $d(a)=a^{-1} a_{[0]} \otimes a_{[1]}=1_{A} \otimes 1_{H}$, then $a_{[0]} \otimes a_{[1]}=a \otimes 1_{H}$, so $a \in B$, and the sequence is exact at $\mathbb{G}_{m}(A)$.

For $X \in G^{i}(A \otimes H)$, let $g(X)=A^{X}$, with notation as in Lemma 2.1. $g$ is multiplicative: take $X=\sum_{i} a_{i} \otimes h_{i}$ and $Y=\sum_{j} b_{j} \otimes k_{j}$ in $G^{i}(A \otimes H)$, then $A^{X} \otimes_{A} A^{Y}=A$ as an $A$ bimodule, with comultiplication given by

$$
\rho_{A^{X} \otimes_{A} A^{Y}}(1)=\sum_{i, j} a_{i} \otimes_{A} b_{j} \otimes h_{i} k_{j}=X Y,
$$

as needed.

If $g(X)=\{A\}$ in $\operatorname{Pic}^{H}(A)$, then there exists an $H$-colinear $A$-linear isomorphism $f: A^{X} \rightarrow A$. Then $f(1)=a$ is invertible in $A$, and, since $f$ is $H$-colinear, $a_{[0]} \otimes a_{[1]}=$ $\rho(a)=(f \otimes H)(X)=a X$, so $X=a^{-1} a_{[0]} \otimes a_{[1]}=d(a)$, and the sequence is also exact at $G^{i}(A \otimes H)$.

The exactness of the sequence at $\operatorname{Pic}^{H}(A)$ follows from Lemma 2.1.

Remark 3.2. Let $H=k \mathbb{Z}$, and let $A$ be a commutative $\mathbb{Z}$-graded $k$-algebra. Then $\operatorname{Pic}^{H}(A)=\operatorname{Pic}_{\mathrm{g}}(A)$, the graded Picard group of $A$, as introduced in [5] (see also [8]). The exact sequence (3.1) reduces to the exact sequence in [5, Proposition 2.1].

The map $d: \mathbb{G}_{m}(A) \stackrel{d}{\rightarrow} G^{i}(A \otimes H)$ is precisely the map $\mathbb{G}_{m}(A) \rightarrow \mathbb{G}_{m}(A \otimes H)$ in the Harrison complex. From Proposition 3.1, we therefore immediately obtain the following corollary.

Corollary 3.3. With $H$ and $A$ as in Proposition 3.1, we have an isomorphism of abelian groups

$$
\operatorname{Pic}^{H}(A) \cong H_{\text {Harr }}^{1}\left(H, A, \mathbb{G}_{m}\right) \text {. }
$$

This is the promised algebraic interpretation of the first Harrison cohomology group. Note that there are no flatness or projectivity assumptions on $H$ or $A$. We have Hilbert 90 as an easy consequence. 
Corollary 3.4 (Hilbert 90). Let $H, A, B$ be as in Proposition 3.1. If $A$ is a faithfully flat $H$-Galois extension of $B$, then we have an isomorphism of abelian groups:

$$
\operatorname{Pic}(A / B) \cong H_{\text {Harr }}^{1}\left(H, A, \mathbb{G}_{m}\right) .
$$

Proof. From the fact that the monoidal categories $\mathcal{M}_{B}$ and $\mathcal{M}_{A}^{H}$ are equivalent, it follows that $\operatorname{Pic}(B) \cong \operatorname{Pic}^{H}(A)$.

Take the exact sequences (2.5) and (3.1), and observe that they fit into a commutative diagram:

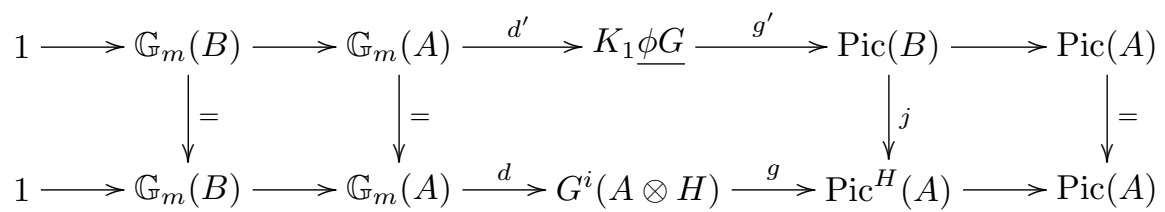

The map $j$ maps $[N] \in \operatorname{Pic}(B)$ to $\left\{N \otimes_{B} A\right\} \in \operatorname{Pic}^{H}(A)$. Using the 'five lemma', we find a map $i: K_{1} \phi G \rightarrow G^{i}(A \otimes H)$.

Lemma 3.5. With notation as above, the maps $i$ and $j$ are injective.

Proof. From the fact that $u$ is a natural transformation between additive endofunctors of the category of $B$-modules, and since $u_{B}$ is an isomorphism, it follows that $u_{N}: N \rightarrow\left(N \otimes_{B} A\right)^{\text {co } H}$ is an isomorphism if $N$ is finitely generated and projective as a $B$-module. So if $N \otimes_{B} A \cong A$, then $N \cong\left(N \otimes_{B} A\right)^{\text {co } H} \cong A^{\text {co } H}=B$, and $j$ is injective. The injectivity of $i$ then follows from an easy diagram-chasing argument.

Our next aim is to characterize the image of $i$. This will be the topic of $\S 4$; it will turn out that we obtain nice results in the case where $H$ is cosemisimple.

\section{Coinvariantly generated relative Hopf modules}

Some of our results will be more specific if we assume that $H$ is a cosemisimple Hopf algebra over a field $k$. Recall that $H$ is cosemisimple if there exists a left integral $\phi$ on $H^{*}$ such that $\phi(1)=1$ (see, for example, [18]). In this case, the coinvariants functor $(\cdot)^{\text {co } H}: \mathcal{M}_{A}^{H} \rightarrow \mathcal{M}_{B}$ is exact (see [15, Lemma 2.4.3]).

A relative Hopf module $M$ is called coinvariantly generated if $c_{M}$ is surjective, or, equivalently, if $M=M^{\mathrm{co} H} A$. If $M$ is coinvariantly generated, and finitely generated as an $A$-module, then we can find a finite set $\left\{m_{1}, \ldots, m_{n}\right\} \in M^{\text {co } H}$ that generates $M$.

It follows immediately from the properties of adjoint functors that $N \otimes_{B} A$ is coinvariantly generated, for every $N \in \mathcal{M}_{B}$; in particular, $A$ is coinvariantly generated. We also have the following lemma.

Lemma 4.1. Let $M \in \mathcal{M}_{A}^{H}$ and $N \in \mathcal{M}_{B}$. If $M$ is an epimorphic image of $N \otimes_{B} A$ in $\mathcal{M}_{A}^{H}$, then $M^{\text {co } H}=0$ implies that $M=0$. 
Proof. If $M^{\mathrm{co} H}=0$, then

$$
\operatorname{Hom}_{A}^{H}\left(N \otimes_{B} A, M\right)=\operatorname{Hom}_{B}\left(N, M^{\text {co } H}\right)=0 .
$$

But $\operatorname{Hom}_{A}^{H}\left(N \otimes_{B} A, M\right)$ contains the epimorphism of relative Hopf modules $N \otimes_{B} A \rightarrow$ $M$, so $M=0$.

If $N$ is an epimorphic image of $M$ in $\mathcal{M}_{A}^{H}$, and if $M$ is coinvariantly generated, then $N$ is also coinvariantly generated.

Lemma 4.2. Assume that $H$ is a cosemisimple Hopf algebra over a field $k$. If $N \in \mathcal{M}_{B}$ is projective, then $N \otimes_{B} A$ is projective in $\mathcal{M}_{A}^{H}$.

Proof. See [7, Proposition 2.5].

Lemma 4.3. Let $k$ be a field.

(1) The forgetful functor $\mathcal{M}_{A}^{H} \rightarrow \mathcal{M}_{A}$ preserves projectives.

(2) If $H$ is cosemisimple, then the forgetful functor also reflects projectivity of finitely generated modules.

Proof. (1) Take $M \in \mathcal{M}_{A}^{H}$ projective, and consider the epimorphism $p: M \otimes A \rightarrow M$, $p(m \otimes a)=m a$ in $\mathcal{M}_{A}^{H}$. The exact sequence

$$
0 \rightarrow \operatorname{Ker} p \rightarrow M \otimes A \stackrel{p}{\rightarrow} M \rightarrow 0
$$

splits in $\mathcal{M}_{A}^{H}$, since $M$ is a projective object, and a fortiori in $\mathcal{M}_{A}$. Hence $M$ is a direct factor of $M \otimes A$, which is a projective $A$-module, so $M$ is also a projective $A$-module.

(2) Let $M$ and $N$ be relative Hopf modules, and assume that $M$ is finitely generated and projective in $\mathcal{M}_{A}$. According to [7, Proposition 4.2], $\operatorname{Hom}_{A}(M, N) \in \mathcal{M}_{A}^{H}$, and it is easy to show that $\operatorname{Hom}_{A}(M, N)^{\operatorname{co} H}=\operatorname{Hom}_{A}^{H}(M, N)$. It follows that the functor $\operatorname{Hom}_{A}^{H}(M,-): \mathcal{M}_{A}^{H} \rightarrow \mathcal{M}$ is exact, since it is the composition of the exact functors $\operatorname{Hom}_{A}(M,-): \mathcal{M}_{A}^{H} \rightarrow \mathcal{M}^{H}\left(M \in \mathcal{M}_{A}\right.$ is projective $)$ and $(\cdot)^{\text {co } H}: \mathcal{M}^{H} \rightarrow \mathcal{M}(H$ is cosemisimple).

Lemma 4.4. Let $H$ be a cosemisimple Hopf algebra over a field $k$, and take $P, Q \in \mathcal{M}_{A}^{H}$ finitely generated as $A$-modules. Assume that $Q$ is a projective object of $\mathcal{M}_{A}^{H}$. Then every epimorphism $f: P \rightarrow Q$ in $\mathcal{M}_{A}^{H}$ has a right inverse in $\mathcal{M}_{A}^{H}$.

Proof. It is clear that $\operatorname{Hom}_{A}(Q, P)$ and $\operatorname{Hom}_{A}(Q, Q)$ are right $H$-comodules, and the map

$$
f^{*}=\operatorname{Hom}_{A}(Q, f): \operatorname{Hom}_{A}(Q, P) \rightarrow \operatorname{Hom}_{A}(Q, Q)
$$

is right $H$-colinear. It follows from Lemma 4.3 that $Q$ is projective as an $A$-module, so $f^{*}$ is surjective. Since $f^{*}$ is $H$-colinear, $f^{*}$ restricts to a surjection

$$
\operatorname{Hom}_{A}^{H}(Q, P)=\operatorname{Hom}_{A}(Q, P)^{\text {co } H} \rightarrow \operatorname{Hom}_{A}^{H}(Q, Q)=\operatorname{Hom}_{A}(Q, Q)^{\text {co } H} .
$$

Take a preimage $g \in \operatorname{Hom}_{A}^{H}(Q, P)$ of the identity map $\operatorname{id}_{Q}$ on $Q$. Then $f \circ g=\mathrm{id}_{Q}$, and the result follows. 
For $M \in \mathcal{M}_{A}$, we will denote the dual module by $M^{*}=\operatorname{Hom}_{A}(M, A)$.

Proposition 4.5. Let $H$ be cosemisimple, and assume that $P \in \mathcal{M}_{A}^{H}$ is coinvariantly generated and finitely generated projective as an A-module. Then

(1) $P^{\mathrm{co} H}$ is a finitely generated projective $B$-module;

(2) $P^{*}$ is coinvariantly generated;

(3) the map $c_{P}$ is an isomorphism in $\mathcal{M}_{A}^{H}$.

Proof. (1) As we have seen, there exist $p_{1}, p_{2}, \ldots, p_{n} \in P^{\mathrm{co} H}$ such that $P=\sum_{i} p_{i} A$. Set $F=A^{n}$ and let $f: F \rightarrow P$ be the $A$-linear map given by $f\left(a_{1}, a_{2}, \ldots, a_{n}\right)=\sum_{i} p_{i} a_{i}$. Then $F \in \mathcal{M}_{A}^{H}$ and $f$ is an epimorphism in $\mathcal{M}_{A}^{H}$. By Lemma 4.4, there exists a monomorphism $g \in \operatorname{Hom}_{A}(P, F)$ such that $f \circ g=\operatorname{id}_{P}$. The restriction of $g$ to $P^{\operatorname{co} H}$ is then a $B$-linear right inverse of the restriction of $f$ to $F^{\text {co } H}$, and $F^{\text {co } H}=B^{n}$, and we obtain (1).

(2) The map $g^{*}=\operatorname{Hom}_{A}(g, A): F^{*} \rightarrow P^{*}$ is surjective and $H$-colinear. The fact that $F^{*}$ is coinvariantly generated then implies that $P^{*}$ is also coinvariantly generated.

(3) Consider the natural transformation $t:(\cdot)^{\operatorname{co} H} \otimes_{B} A \rightarrow(\cdot)$ given by

$$
t_{P}: P^{\mathrm{co} H} \otimes_{B} A \rightarrow P, \quad t_{P}(p \otimes a)=p a .
$$

The map $t_{A}$ is an isomorphism, so $t_{F}$ is an isomorphism by additivity. It follows that $t_{P}$ is an isomorphism, since $F=P \oplus \operatorname{Ker} f$ as $H$-comodules.

Let $X=\sum_{i} a_{i} \otimes h_{i} \in G(A \otimes H)$, and write

$$
A_{X}=\left\{a \in A \mid \rho(a)=a X=\sum_{i} a a_{i} \otimes h_{i}\right\}
$$

and

$$
A_{X}^{i}=\left\{a \in A_{X} \mid a \text { is invertible }\right\} .
$$

Observe that

$$
\operatorname{Im}(d)=\left\{X \in G^{i}(A \otimes H) \mid A_{X}^{i} \neq \emptyset\right\}
$$

and

$$
A_{1 \otimes 1}=A^{\mathrm{co} H} .
$$

Furthermore, $A_{X} A_{Y} \subset A_{X Y}$ : take $a \in A_{X}$ and $b \in A_{Y}$, then $\rho(a)=a X=\sum_{i} a a_{i} \otimes h_{i}$, $\rho(b)=b Y=\sum_{j} b b_{j} \otimes k_{j}$ and

$$
\rho(a b)=a_{[0]} b_{[0]} \otimes a_{[1]} b_{[1]}=\sum_{i, j} a a_{i} b b_{j} \otimes h_{i} k_{j}=a b X Y .
$$

Also $A_{X}^{i} \cap A_{Y}^{i}=\emptyset$ if $X \neq Y$. 
Lemma 4.6. The set

$$
E=\left\{X \in G^{i}(A \otimes H) \mid A A_{X}=A \text { and } A A_{X^{-1}}=A\right\}
$$

is a subgroup of $G^{i}(A \otimes H)$ containing $\operatorname{Im}(d)$.

Proof. If $X \in \operatorname{Im}(d)$, then there exists an invertible $a \in A_{X}$, and then $A A_{X}=A$. Since $X^{-1} \in \operatorname{Im}(d)$, we also have $A A_{X^{-1}}=A$, hence $X \in E$. It is clear that $1 \otimes 1 \in E$. If $X, Y \in E$, then $A A_{X Y} \supset A A_{X} A_{Y}=A A_{Y}=A$, and, in a similar way, $A A_{(X Y)^{-1}}=A$, hence $X Y \in E$. Finally, if $X \in E$, then obviously $X^{-1} \in E$.

Proposition 4.7. Consider the injective map $j: \operatorname{Pic}(B) \rightarrow \operatorname{Pic}^{H}(A)$. If $H$ is a cosemisimple Hopf algebra over a field $k$, then

$$
\operatorname{Im}(j)=\left\{\{M\} \in \operatorname{Pic}^{H}(A) \mid M \text { is coinvariantly generated }\right\} .
$$

Proof. $M \otimes_{B} A$ is coinvariantly generated, so $\operatorname{Im}(j)$ is contained in the desired set. If $H$ is cosemisimple, and $\{N\} \in \operatorname{Pic}^{H}(A)$, with $N$ coinvariantly generated, then $N=\left(N^{\mathrm{co} H}\right) \otimes_{B} A \in \operatorname{Im}(j)$, by Proposition 4.5 (3).

Lemma 4.8. Take $X \in G^{i}(A \otimes H)$. Then $A^{X}$ is coinvariantly generated if and only if $A A_{X^{-1}}=A$. If $H$ is cosemisimple, then this is also equivalent to $X \in E$.

Proof. The first statement follows from the fact that $\left(A^{X}\right)^{\text {co } H}=A_{X^{-1}}$. Indeed, $a \in\left(A^{X}\right)^{\text {co } H}$ if and only if $\rho_{X}(a)=X a=a \otimes 1$, if and only if $\rho(a)=(1 \otimes 1) a=$ $X^{-1}(a \otimes 1)=a X^{-1}$, which means that $a \in A_{X^{-1}}$.

Let $H$ be cosemisimple. Note that $\left(A^{X}\right)^{*} \cong A^{X^{-1}}$ as relative Hopf modules. If $A^{X}$ is coinvariantly generated, then so is $A^{X^{-1}}$, by Proposition 4.5, and then $X \in E$.

Now we are able to prove the main result of this section.

Theorem 4.9. Consider the monomorphism $i: K_{1} \phi G \rightarrow G^{i}(A \otimes H)$ introduced in Lemma 3.5.

Then $\operatorname{Im}(i) \subset E$ and $\operatorname{Im}(i)=E$ if $H$ is a cosemisimple Hopf algebra over a field $k$. In this situation, $\operatorname{Pic}(A / B) \cong E$.

Proof. Take $[(M, \alpha)] \in K_{0} \underline{\psi G}$, and let $i[(M, \alpha)]=X \in G^{i}(A \otimes H)$. Then

$$
\left\{A^{X}\right\}=j\left(g^{\prime}[(M, \alpha)]\right)=j([M])=\left\{M \otimes_{B} A\right\},
$$

hence $A^{X}$ is coinvariantly generated and $A A_{X^{-1}}=A$, by Lemma 4.8. In a similar way, $i\left([(M, \alpha)]^{-1}\right)=X^{-1}$, and $A^{X^{-1}} \cong M^{*} \otimes_{B} A$ is coinvariantly generated, so $A A_{X}=A$, again by Lemma 4.8. This proves that $X \in E$.

Assume now that $H$ is cosemisimple, and take $X \in E$. It follows from Lemma 4.8 that $A^{X}$ is coinvariantly generated, and from Proposition 4.7 that $A^{X}=M \otimes_{B} A$ for some $M \in \underline{\operatorname{Pic}}(B)$. Since the image of $M$ in $\operatorname{Pic}(A)$ is trivial, $[M]=g^{\prime}[(M, \alpha)]$ for some $(M, \alpha) \in \mathcal{C}$. Write $i[(M, \alpha)]=Y$. Then $X=Y d(a)$, for some $a \in \mathbb{G}_{m}(A)$. Consider the map $\alpha^{\prime}: M \otimes_{B} A \rightarrow A, \alpha^{\prime}(m \otimes b)=a^{-1} \alpha(m \otimes b)$. Then $i\left[\left(M, \alpha^{\prime}\right)\right]=X$. 


\section{On the group-like elements}

We have an injective map $i: G(H) \rightarrow G(A \otimes H), i(g)=1_{A} \otimes g$. Everything simplifies if $i$ is an isomorphism. We discuss two situations in which this is (almost) the case.

Recall that a commutative algebra which is an integral domain is called normal if it is integrally closed in its field of fractions.

Proposition 5.1. Let $k$ be an algebraically closed field, let $A$ be a finitely generated commutative normal $k$-algebra and let $G$ be a connected algebraic group acting rationally on $A$. Let $H$ be the affine coordinate ring of $G$, and $\chi(G)$ be the group of characters of $G$. Then

$$
G(A \otimes H)=\{1 \otimes \phi \mid \phi \in G(H)=\chi(G)\} .
$$

Proof. Let $x=\sum_{i} a_{i} \otimes f_{i} \in G(A \otimes H)$. Then we have

$$
\sum_{i}\left(a_{i} \otimes f_{i(1)} \otimes f_{i(2)}\right)=\sum_{i, j}\left(a_{i} a_{j[0]} \otimes\left(f_{i} * a_{j[1]}\right) \otimes f_{j}\right)
$$

and $\sum a_{i} \varepsilon\left(f_{i}\right)=1$. The map

$$
\alpha: A \otimes H \rightarrow \operatorname{Hom}(k G, A), \quad \alpha(a \otimes f)(g)=a f(g)
$$

is injective. Let $\phi=\alpha(x)$. Using (5.1), we compute for all $g, g^{\prime} \in G$ that

$$
\begin{aligned}
\phi\left(g g^{\prime}\right) & =\sum_{i} a_{i} f_{i}\left(g g^{\prime}\right)=\sum_{i} a_{i} f_{i(1)}(g) f_{i(2)}\left(g^{\prime}\right) \\
& =\sum_{i, j} a_{i} a_{j[0]}\left(\left(f_{i} * a_{j[1]}\right)(g)\right) f_{j}\left(g^{\prime}\right) \\
& =\sum_{i, j} a_{i} a_{j[0]} f_{i}(g) a_{j[1]}(g) f_{j}\left(g^{\prime}\right) \\
& =\sum_{i, j}\left(g \cdot a_{j}\right) f_{j}\left(g^{\prime}\right) a_{i} f_{i}(g) \\
& =\sum_{i, j} g \cdot\left(a_{j} f_{j}\left(g^{\prime}\right)\right) a_{i} f_{i}(g) \\
& =\left(g \cdot\left(\phi\left(g^{\prime}\right)\right)\right) \phi(g) .
\end{aligned}
$$

From the second equality, we have $1=\sum_{i} a_{i} f_{i}\left(1_{G}\right)=\phi\left(1_{G}\right)$. For every $g \in G, \phi(g)$ is invertible in $A$, with inverse $g \cdot\left(\phi\left(g^{-1}\right)\right)$. By the proof of [13, Proposition 1b, p. 46], $\phi(g) \in k$ for every $g \in G$, so $\phi \in \chi(G)$. Now $\chi(G)=G(H) \subset H$ (see $[\mathbf{1 2}$, p. 25]), so it follows in particular that $\phi \in H$. For all $g \in G$ we now have that

$$
\alpha(1 \otimes \phi)(g)=\phi(g)=\sum_{i} a_{i} f_{i}(g)=\alpha(x)(g),
$$

hence $x=1 \otimes \phi$, by the injectivity of $\alpha$. 
Now consider the situation from Remark 3.2: $H=k \mathbb{Z} \cong k\left[X, X^{-1}\right]$, and $A$ is a commutative $\mathbb{Z}$-graded algebra. In this situation $A \otimes H=A \otimes k\left[X, X^{-1}\right]$. Group-like elements in $A \otimes H$ can be constructed as follows. Let $1=e_{1}+\cdots+e_{n}$ with the $e_{i}$ orthogonal idempotents, and take $d_{1}, \ldots, d_{n} \in \mathbb{Z}$. Then $\sum_{i=1}^{n} e_{i} \otimes X^{d_{i}}$ is a group-like element in $A \otimes k\left[X, X^{-1}\right]$. In this way, we have an embedding of $\mathcal{C}(\operatorname{Spec}(A), \mathbb{Z})$, the continuous functions from $\operatorname{Spec}(A)$ (with the Zariski topology) to $\mathbb{Z}$ (with the discrete topology), into $G\left(A \otimes k\left[X, X^{-1}\right]\right)$. The first author was amazed to see that one of his first results, [5, Theorem 2.3], can be restated in such a way that it becomes a result about corings. Recall that a commutative ring is called reduced if it has no non-trivial nilpotents.

Proposition 5.2. Let $A$ be a reduced $\mathbb{Z}$-graded commutative $k$-algebra. Then the $\operatorname{map} \mathcal{C}(\operatorname{Spec}(A), \mathbb{Z}) \rightarrow G\left(A \otimes k\left[X, X^{-1}\right]\right)$ is a bijection.

Example 5.3 (cf. Example 2.6 in [5]). Proposition 5.2 does not hold if $A$ contains nilpotent elements; this is related to the fact that there exist non-homogeneous units in this situation. Let $A=k[x]$, with $x^{2}=0$, and put a $\mathbb{Z}$-grading on $A$ by taking $\operatorname{deg}(x)=1$. Then $1+a x \in \mathbb{G}_{m}(A)$, and $d(1+a x)=(1-a x) \otimes 1+a x \otimes X$ is a group-like element in $G\left(A \otimes k\left[X, X^{-1}\right]\right)$ which is not in the image of $\mathcal{C}(\operatorname{Spec}(A), \mathbb{Z})$.

Acknowledgements. The research herein was supported by the project G.0278.01 'Construction and applications of non-commutative geometry: from algebra to physics' from FWO Vlaanderen.

\section{References}

1. H. BAss, Algebraic K-theory (Benjamin, New York, 1968).

2. T. BRZEZIŃSKI, The structure of corings, Induction functors, Maschke-type theorem, and Frobenius and Galois properties, Alg. Representat. Theory 5 (2002), 389-410.

3. T. BRzeziński, The structure of corings with a group-like element, Banach Center Publicat. 61 (2003), 21-35.

4. T. BRZEZiński AND R. WisBauer, Corings and comodules, London Mathematical Society Lecture Note Series, vol. 309 (Cambridge University Press, 2003).

5. S. CAentePEel, A cohomological interpretation of the graded Brauer group, I, Commun. Alg. 11 (1983), 2129-2149.

6. S. CAentePEel, Brauer groups, Hopf algebras and Galois theory, K-Monographs in Mathematics, vol. 4 (Kluwer, 1998).

7. S. CAenePeEl And T. GuÉDÉnon, Projectivity of a relative Hopf module over the subring of coinvariants, in Hopf algebras (ed. J. Bergen, S. Catoiu and W. Chin), pp. 97-108, Lecture Notes in Pure and Applied Mathematics, vol. 237 (Marcel Dekker, New York, 2004).

8. S. Caenepeel and F. VAn Oystaeyen, Brauer groups and the cohomology of graded rings, Monographs and Textbooks in Pure and Applied Mathematics, vol. 121 (Marcel Dekker, New York, 1988).

9. F. DeMeyer and E. Ingraham, Separable algebras over commutative rings, Lecture Notes in Mathematics, vol. 181 (Springer, 1971).

10. S. DǍsCǍlescu, C. NǍstǎsescu AND Ş. Raianu, Hopf algebras: an introduction, Monographs and Textbooks in Pure and Applied Mathematics, vol. 235 (Marcel Dekker, New York, 2001).

11. L. El Kaoutit, J. Gómez-Torrecillas and F. J. Lobillo, Semisimple corings, Algebra Colloq. 11 (2004), 427-442. 
12. J. C. JAntzen, Representations of algebraic groups, Pure and Applied Mathematics, vol. 131 (Academic, 1987).

13. A. Magid, Finite generation of class groups of rings of invariants, Proc. Am. Math. Soc. 60 (1976), 45-48.

14. A. Magid, Picard groups of rings of invariants, J. Pure Appl. Alg. 17 (1980), 305-311.

15. S. Montgomery, Hopf algebras and their actions on rings (American Mathematical Society, Providence, RI, 1993).

16. M. E. SweEdler, Cohomology of algebras over Hopf algebras, Trans. Am. Math. Soc. 133 (1968), 205-239.

17. M. E. SweEdLeR, Hopf algebras (Benjamin, New York, 1969).

18. M. E. SweEdLer, The predual Theorem to the Jacobson-Bourbaki theorem, Trans. Am. Math. Soc. 213 (1975), 391-406. 